\title{
Development of a Fully-Integrated Ultrasensitive Wireless Sensor Utilizing Carbon Nanotubes and Surface Plasmon Theory
}

\author{
Trang T. Thai ${ }^{(1)}$, Amil Haque ${ }^{(1)}$, Justin Ratner ${ }^{(1)}$, Gerald R. DeJean $^{(2)}$, and Manos M. Tentzeris ${ }^{(1)}$ \\ (1) GEDC, School of ECE, Georgia Institute of Technology, Atlanta, GA 30332, U.S.A. \\ (2) Microsoft Research, One Microsoft Way, Redmond, WA 98052, U.S.A. \\ Email: trang.thai@gatech.edu
}

\begin{abstract}
A practical application of surface plasmon resonance (SPR) for the realization of an ultrasensitive, fully-integrated, fully-packaged wireless sensor, that can operate in microwave frequencies based on the gas sensitivity of carbon nanotube (CNT) mixtures, is proposed. The sensor consists of a corrugated aluminum plate whose surface is periodically covered with a thin layer of the CNT materials. The incident TM-polarized waves on this surface excite the surface plasmon (SP) mode, thus resulting in a drop of power of the reflected wave. The change in the electrical properties of the CNT mixtures when exposed to gases results in the change of the SPR observable in the reflected waves. For the first time, a CNT-based wireless sensor has been designed to show a frequency shift of $400 \mathrm{MHz}$ while operating in the $60 \mathrm{GHz}$ range.
\end{abstract}

\section{Introduction}

In the current gas sensing technology, most gas sensor devices require some form of a direct physical contact. CNT mixtures/composites were found to have electrical properties highly sensitive to extremely small quantities of gases, such as ammonia $\left(\mathrm{NH}_{3}\right)$, carbon dioxide $\left(\mathrm{CO}_{2}\right)$, nitrogen oxide $(\mathrm{NOx})$, etc. at room temperatures with a very fast response time [1]. Most CNT-based active sensors employ the change in resistance (easily affected by moisture), thermoelectric power, electrical breakdown voltage, or dielectric properties of the nanotubes for the gas detection. Many of these sensors also require a high temperature for operating conditions. Therefore, a gas sensor that can operate at room temperature while maintaining a high sensitivity is highly desirable. Due to the strong demand for monitoring hazardous gases in remote or rugged environments, integration of the sensors with wireless monitoring technology is also desired for realtime remote sensing. While many types of direct physical contact sensors that utilize sensing properties of CNTs have been proposed, there has been very limited development on CNT-based wireless sensors due to the fact that the change of their dielectric constant when the sensing CNT mixtures/composites are exposed to gas is around 1-4 \% [1][2]. Chopra et al. demonstrated a resonator-based gas sensor that enables remote sensing in which the resonator is coated with CNT composite [2]. The operation is based on the change of the effective relative permittivity of the resonator which results in a resonant frequency shift of 1-4 $\mathrm{MHz}$ when exposed to different gases including $\mathrm{NH}_{3}$ and $\mathrm{CO}_{2}$ at several hundreds ppb (parts per billion) to several ppm (parts per million) for a center resonant frequency of $3.889 \mathrm{GHz}$.
However, this shift is very small, and this method does not support further miniaturization.

This paper introduces a practical application of surface plasmon resonance (SPR) for the realization of an ultrasensitive, fully-integrated, fully-packaged wireless sensor that can operate in microwave frequencies. SPR is a chargedensity oscillation that may be excited by incoming electromagnetic waves, and exists on the surface of two media with dielectric constants of opposite signs such as a metal and a dielectric. SPR occurs under conditions of total internal reflection with p-polarized light (TM-polarized waves). Since the propagation constant of surface plasmon waves (SPWs) in the metal layer is always higher than that of the wave propagation in the dielectric layer, the momentum of the incident wave has to be enhanced to match that of the SPW to achieve SPR. This is usually done by using total reflection in prism couplers or optical waveguides or by using diffraction at the surface of diffraction gratings where the periodic corrugation provides the necessary in-plane wave-vector enhancement in multiples of the grating vector. The working of the latter approach in coupling the incident wave to the SPW has been demonstrated for both optical and microwave incoming waves [4][5]. Since SPWs strongly depend on the interface conditions, their resonance condition is very sensitive to variations in the electrical properties of the dielectric adjacent to the metal layer that supports SPWs (noble metals, such as gold, silver, copper). Therefore, we propose to use CNT mixtures/composites as the dielectric thin-film on an aluminum plate to realize a highly sensitive gas sensor that operates based on surface plasmons whose resonant frequency is determined by the air/aluminum/CNT dielectric interface.

\section{Principles of Operation and Modeling of the Carbon Nanotube based Material}

The surface plasmons (SPs) are the coherent fluctuations of electron charges on a metal-to-dielectric boundary [6]. The surface mode propagates as a longitudinal wave along the interface the field vectors of which reach their maxima at the interface and decay evanescently into both media. Since the propagation constant of SPWs in the metal layer is always higher than that of the wave propagation in the dielectric layer, the momentum of the incident wave has to be enhanced to match that of the SPW to achieve SPR. In this paper, we employ the grating technique to couple the microwave incident waves to SPs on the aluminum surface [4]. 
Using Agilent Design Systems (ADS) and HFSS by Ansoft Corp., we obtain the permittivity model for the dielectric that represents the CNT mixtures implemented by Dragoman et. al. [8][9]. The dielectric constant values are 4.30 and 2.85 at $10 \mathrm{GHz}$ and $60 \mathrm{GHz}$ respectively. In the first part of study, the objective is to observe the effect of small changes in dielectric constant on the SPR observed in a rectangular grating with a lossless dielectric on its surface. Therefore the dielectric is set to lossless and a change of $+2 \%$ is applied to the dielectric constant in order to model the reacted sample in set up shown in Fig.1. In the second part of the study (Fig. 4), the reacted sample is modeled with dielectric constant of 1.90 and loss tangent to be 0.698 (deduced from [8]).

\section{Simulations and Results}

\section{A. Sensing based on enhancement of SPR in transmission}

The detection performance using SPR for a small change of dielectric constant $(2 \%)$ is investigated using the structure shown in Fig. 1, where the metal is aluminum and the CNT composite represents the dielectric. In this set up which is advanced from Akarca-Biyikli's study [7], h1 $(13.9 \mathrm{~cm})$ is the distance from the transmitter waveport to the slit, and h2 $(29.1 \mathrm{~cm})$ is the distance from the slit to the receiver waveport. The cross section of the corrugated sample is shown in Fig. 2. The dimensions of the structure are as follows: $a=2 \mathrm{~mm}, \mathrm{~b}=$ $4 \mathrm{~mm}, \mathrm{t}=\mathrm{g}=\mathrm{h}=4 \mathrm{~mm}$, and $\lambda_{\mathrm{g}}=4 \mathrm{~mm}$. In this set up $\varepsilon_{\mathrm{r}}$, is set to 4.30 in the absence of the gas, and $\varepsilon_{\mathrm{r}}$, is set to 4.386 in the presence of the gas; $\varepsilon_{\mathrm{r}}$, is set to zero for lossless assumption. The simulation is performed in FDTD Solutions (evaluation version) by Lumerical Solutions, Inc. TM-polarized waves are incident on the corrugated metal plate covered with a CNT material layer of thickness $t$. The transmission of this power through the subwavelength slit is collected by a receiver on the other side of the metal plate as shown in Fig. 1. Simulation results are shown in Fig. 3 for three cases: aluminum plate covered by dielectric layer with no grating in the absence of gas, aluminum plate covered by dielectric layer with grating in the absence of gas, and aluminum plate covered by dielectric layer with grating in the presence of gas. The results show a sharp peak of enhancement around 22 $\mathrm{GHz}$ in comparison to the non-corrugated structure (case a in Fig. 3) and the corrugated one in the absence of gas (case b in Fig. 3). In the absence of gas, the enhanced peak is at 22.6 $\mathrm{GHz}$ with a magnitude of 0.218 . In the presence of gas, this peak is shifted to $22.2 \mathrm{GHz}$ with a magnitude of 0.208 . The frequency shift is about $400 \mathrm{MHz}$. This result successfully demonstrates our proof-of-concept on the use of SPR for gas sensing with extremely small change $(2 \%)$ in the dielectric constant of the sensing material.

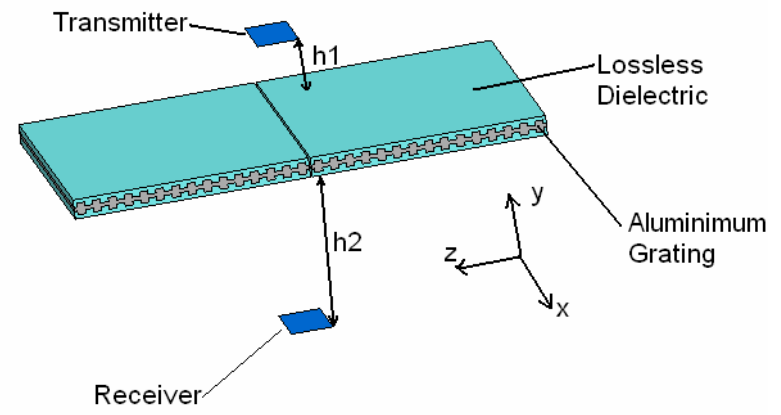

Fig. 1. Simulation set up for sensing using the enhancement of SPR in transmission with $2 \%$ change in the sensing dielectric represents CNT composite

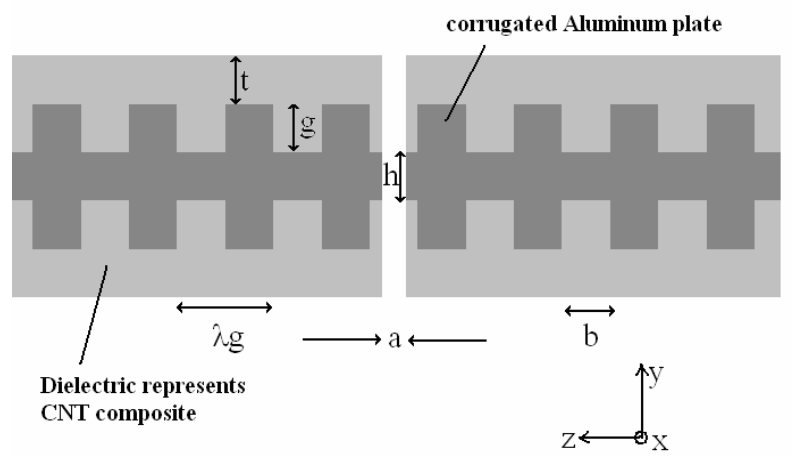

Fig. 2. Cross section of the slotted sample with grating in transmission set up

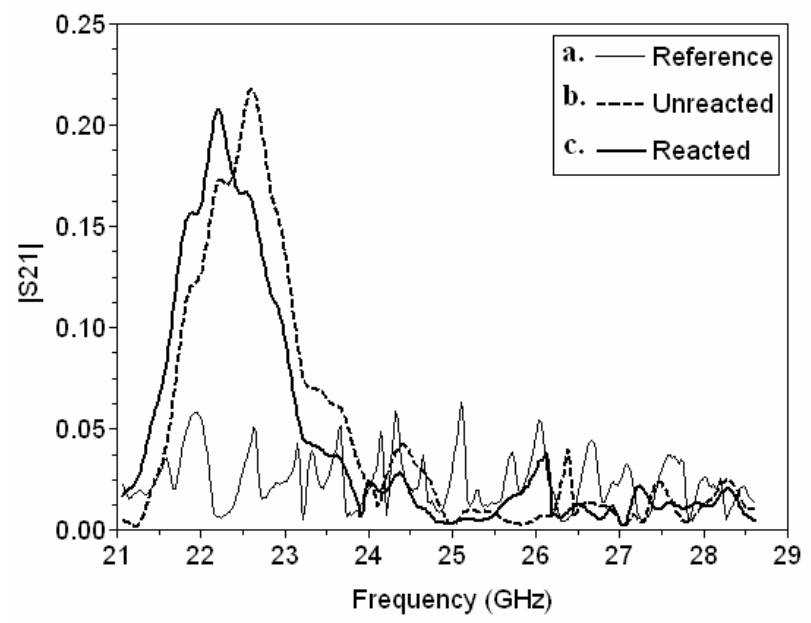

Fig. 3. Magnitude of the transmission through the subwavelength slit plotted against frequency for a) the reference simulation of non-corrugated plate covered by unreacted dielectric layer, b) the simulation of corrugated plate covered with un-reacted dielectric layer, and c) the simulation of corrugated plate covered with reacted dielectric layer.

\section{B. Sensing based on minimum reflection due to SPR}

Illustration of the reflection set up for the gas sensor is shown in Fig. 4. This set up is simulated in HFSS software by Ansoft Corp. The parameters that model the CNT sensing 
material in this set up are deduced from Dragoman et. al. work [8][9] as discussed in section II. The DWNTs is placed on top of the corrugation as shown in Fig. 5. In this structure, $\mathrm{h}=0.8 \mathrm{~mm}, \mathrm{t}=0.2 \mathrm{~mm}, \mathrm{~g}=0.7 \mathrm{~mm}, \mathrm{~b}=1.75 \mathrm{~mm}, \theta=30^{\circ}$, and $\lambda_{\mathrm{g}}=3.5 \mathrm{~mm}$. The length of the aluminum plate implemented in simulation is $11 \lambda_{\mathrm{g}}$, and the length in the $\mathrm{x}$-direction of the metal plate is $42 \mathrm{~mm}$. The DWNTs are modeled as a dielectric material with the real part $\left(\varepsilon_{\mathrm{r}}{ }^{\prime}\right)$ and imaginary part $\left(\varepsilon_{\mathrm{r}}{ }^{\prime \prime}\right)$ of the permittivity set to 2.845 and 2.176 , respectively in the absence of $\mathrm{N}_{2}$, and 1.9 and 1.326 , respectively in the presence of $\mathrm{N}_{2}$ [8][9]. We performed simulations to observe the reflection level of 4 different samples as shown in Fig. 5 for comparison. The reflectance of the four different samples in the frequency range $40-80 \mathrm{GHz}$ is shown in Fig. 6 .

The SPR is easily observed between the non-corrugated sample (Fig. 5a) and corrugated samples (Fig. 5b, 5c, 5d) where the reflectivity is decreased sharply starting at around $50 \mathrm{GHz}$. The periodic minima observed for frequencies above $50 \mathrm{GHz}$ are due to the fact that a multiple integer of the SPR wavelength can also excite higher orders of the SP modes explained in grating theory. The minima of the frequencies are shifted as expected between samples covered with dielectric (Fig. 5c and 5d) and samples without dielectric (Fig. $5 b$ ). In the absence and presence of $\mathrm{N}_{2}$, the frequency shift is observed to be approximately $400 \mathrm{MHz}$ and the amplitude is changed from 0.08 to approximately 0.11 between the nonreacted and reacted cases with $\mathrm{N}_{2}$ being used in the DWNT mixture.

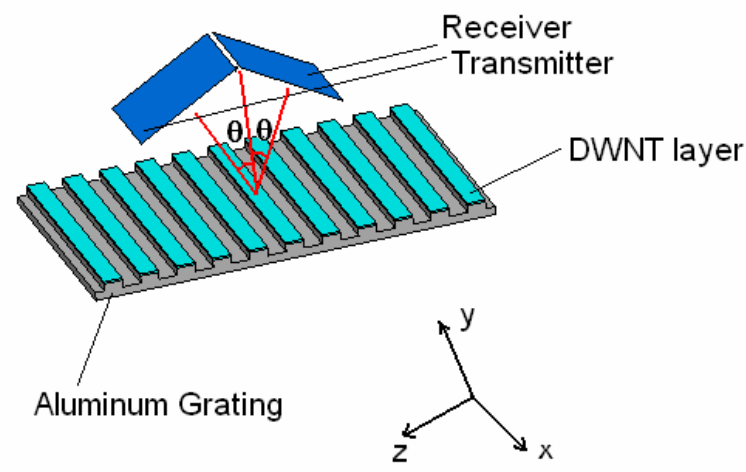

Fig. 4. Detection of reflection set up for simulation of the corrugated Aluminum plate with covered periodically by dielectric represents CNT mixture b.

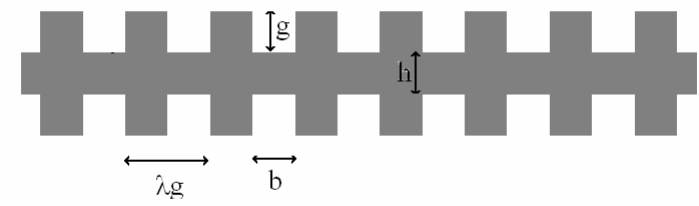

c.

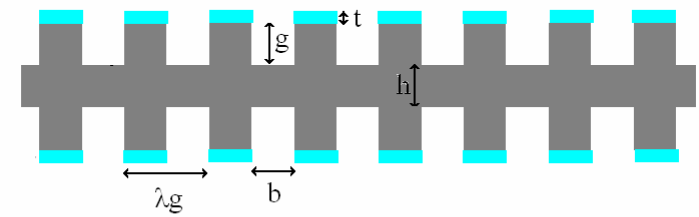

d.

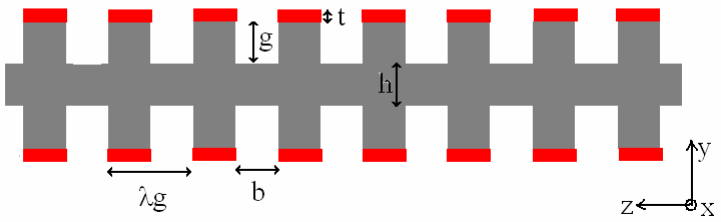

Fig. 5. Different samples implemented in simulation for reflectance comparison for a) flat aluminum plate, b) corrugated aluminum plate, c) corrugated aluminum plate with periodically covered dielectric represents unreacted DWNTs (absence of $\mathrm{N}_{2}$ ), and d) corrugated aluminum plate with periodically covered dielectric represents reacted DWNTs (presence of $\mathrm{N}_{2}$ ).

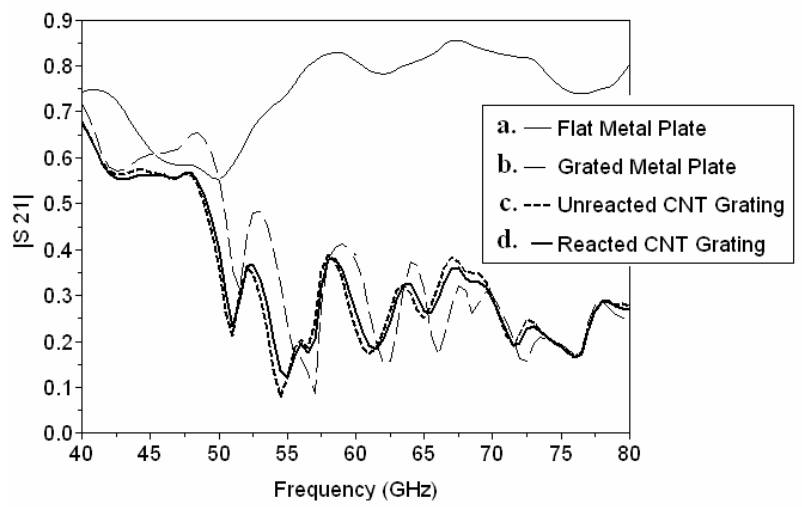

Fig. 6. Magnitude of $S 21$ in the reflection set up with angle $\theta$ equals to $30^{\circ}$ for reflectivity of a) flat aluminum plate, b) corrugated aluminum plate, c) corrugated aluminum plate with periodically covered dielectric represents DWNTs in the absence of $\mathrm{N}_{2}$, and d) corrugated aluminum plate with periodically covered dielectric represents DWNTs in the presence of $\mathrm{N}_{2}$.

\section{Conclusions}

We have presented a novel approach for sensing gas in real time with capabilities of remote sensing in a microwave wireless network. Our design demonstrated a practical application of surface plasmon resonance (SPR) for the realization of an ultrasensitive, fully-integrated, fullypackaged wireless sensor utilizing the ultra sensitive properties of the CNT materials to detect different gases. 
Based on the shift in SPR and the level of reflectivity, selectivity in sensing can also be achieved. The SPR approach also allows further miniaturization as the size of the sensor is decreased when the operating frequency is increased without affecting the sensitivity of the sensor. Although the recovery time of the CNT composites is slow (6-10 hrs) which is the limitation of all gas sensors based on CNTs, the response time is very fast within a few minutes. This feature together with high sensitivity and remote sensing capability may allow detection of cracking in spacecraft in real-time (based on detection of gas leakage through the cracks), or monitoring the leakage of dangerous gases into the public and private environments.

\section{Acknowledgments}

The authors would like to acknowledge the support of Georgia Electronic Design Center.

\section{References}

1. K. G. Ong, K. Zeng, C. A. Grimes, "A wireless, passive carbon nanotube-based gas sensor," IEEE Sens. J., 2, pp 82-88 (2002).

2. S. Chopra, A.Pham, J. Gaillard, A. Parker, A. M. Rao, "Carbon-nanotube-based resonant-circuit sensor for ammonia," Appl. Phys. Lett. 80, 4632 (2002).

3. H Raether, Surface plasmons on smooth and rough surfaces and on gratings, Springer-Verlag, Berlin, 1988.

4. A. P. Hibbins, J. R. Sambles, C. R. Lawrence, "Gratingcoupled surface plasmons at microwave frequencies," J. App. Phys., Vol 86, Issue 4, 15 Aug 1999.

5. Ji Homola, Ivo Koudela, Sinclair S. Yee, "Surface plasmon resonance sensors based on diffraction gratings and prism couplers: sensitivity comparison," Sensors and Actuators B: Chem, Vol 54, Issues 1-2, 25, pp 16-24, Jan 1999.

6. R. H. Ritchie, "Plasma losses by fast electrons in thin films," Phys. Rev. 106, 874 (1957).

7. S. S. Akarca-Biyikli, I. Bulu, and E. Ozbay, "Resonant excitation of surface plasmons in one-dimensional metallic grating structures at microwave frequencies," J. Opt. A: Pure Appl. Opt., 7, (2005) 159-164.

8. M. Dragoman, K. Grenier, D. Dubuc, L. Bary, R. Plana, E. Fourn, E. Flahaut, "Millimeter wave carbon nanotube gas sensor," App. Phy. Lett. 101, 106103 (2007).

9. M. Dragoman, K. Grenier, D. Dubuc, L, Bary, E. Fourn, and R. Plana, " Experimental determination of microwave attenuation and electrical permittivity of double-walled carbon nanotubes,” App. Phys. Lett. 88, 153108 (2006). 